\title{
OPTIMIZACIÓN DE LA PRODUCTIVIDAD EN INDUSTRIAS PESQUERAS
}

\section{PRODUCTIVITY OPTIMIZATION IN FISHING INDUSTRIES}

\author{
López-Anchundia Eduardo ${ }^{1 *}$; Santana-Pachay Gonzalo²; Reyes-Solórzano Javier³ \\ ${ }^{1}$ Universidad Técnica de Manabí, UTM. Portoviejo, Ecuador. \\ ${ }^{2}$ Universidad Laica Eloy Alfaro de Manabí, ULEAM. Manta, Ecuador. \\ ${ }^{3}$ Docente de la Universidad Laica Eloy Alfaro de Manabí, ULEAM. Manta, Ecuador.
}

*Correo: egla-bioq10@gmail.com

\begin{abstract}
Resumen
Las industrias pesqueras son una base importante de la economía del país y constituyen un importante segmento de las exportaciones, puesto que el $99 \%$ de las exportaciones que realizan las industrias pesqueras tienen como destino países europeos, norteamericanos y sudamericanos. Es evidente el gasto indiscriminado de agua que durante la etapa de lavado de parrillas con pescado clasificado y congelado se genera en las plantas pesqueras, asimismo es posible fomentar una cultura de ahorro de energía eléctrica. Otro suceso importante que se da en esta área de proceso es la mala distribución y demanda innecesaria del recurso humano. Es por esta razón que este artículo tiene como objetivo analizar procesos productivos de las plantas atuneras y sus respectivos puntos de mejora para disminuir el consumo de recursos, considerando los principios del sistema HACCP. La mejora permanente y renovadora de la calidad de los procesos productivos de las industrias pesqueras debe ser un principio que forme parte de la política de calidad, manejo ambiental y seguridad de los trabajadores. Sin embargo, la optimización de los procesos implica cambios en los procesos productivos, los cuales deben ser valorados en el sistema HACCP implantado en gran parte de las industrias pesqueras.
\end{abstract}

Palabras clave: industria, optimización, mejora continua, HACCP, producción pesquera.

\begin{abstract}
The fishing industries are an important base of the country's economy and constitute an important segment of exports, since $99 \%$ of the exports made by the fishing industries are destined for European, North American and South American countries. It is evident the indiscriminate use of water that is generated in fishing plants during the grilling stage with frozen and classified fish, it is also possible to promote a culture of saving electrical energy. Another important event that occurs in this process area is the poor distribution and unnecessary demand for human resources. It is for this reason that this article aims to analyze the productive processes of tuna plants and their respective points of improvement to decrease the consumption of resources, considering the principles of the HACCP system. The permanent and renovating improvement of the quality of the productive processes of the fishing industries must be a principle that is part of the quality, environmental management and worker safety policy. However, the optimization of processes implies changes in production processes, which must be valued in the HACCP system implemented in a large part of the fishing industries.
\end{abstract}

Keywords: industry, optimization, continuous improvement, HACCP, fish production.

Información del manuscrito:

Fecha de recepción: 04 de abril de 2017

Fecha de aceptación: 29 de junio de 2017

Fecha de publicación: 10 de julio de 2017 


\section{Introducción}

Las industrias pesqueras son una base importante de la economía del país y constituyen un importante segmento de las exportaciones, puesto que el $99 \%$ de las exportaciones que realizan las industrias pesqueras tienen como destino países europeos, norteamericanos y sudamericanos. Estas industrias buscan mejorar constantemente la calidad de sus productos y gestionan sus procesos bajo principios de innovación y mejora continua, provocando que con el paso de los años se han ido modernizando las plantas industriales mediante la implementación y acondicionamiento de las áreas productivas (Barclay \& Cartwright, 2007).

Comúnmente las variedades procesadas en las industrias pesqueras de la ciudad de Manta son skipjack, Yellowfin, Bigeye, albacora y melva. Se producen lomos precocinados en fundas termoencogibles, conservas (latas y Pouch) en varios formatos de peso $y$ atendiendo los requerimientos específicos de cada cliente. Es bien conocido que las plantas atuneras registran excesivos consumos de recursos como agua, energía Eléctrica y mano de obra (Barclay \& Cartwright, 2007).

De alguna $u$ otra forma el uso excesivo de los recursos no se percibe a simple vista, y se han advertido las deficiencias que se presentan en las parrillas, debido al maltrato al que se exponen durante el proceso, sin dejar de lado que en ocasiones esporádicas se ha optado por colocar las parrillas en el piso, generando contaminación de las mismas; pues una auditoría nacional o internacional podría exponer a sanciones 0 incluso al cierre parcial o total de la planta.

Es evidente el gasto indiscriminado de agua, que durante la etapa de lavado de parrillas con pescado clasificado y congelado, se genera en las plantas pesqueras, asimismo es posible fomentar una cultura de ahorro de energía eléctrica. Otro suceso importante que se da en esta área de proceso es la mala distribución y demanda innecesaria del recurso humano que se ha visto en ocasiones afectada por el ausentismo al que en determinados momentos se exponen estas empresas (Ruiz \& Karim, 2016). Es 
por esta razón que este artículo tiene como objetivo analizar procesos productivos de las plantas atuneras y sus respectivos puntos de mejora para disminuir el consumo de recursos, considerando los principios del sistema HACCP.

\section{Sistema de análisis de peligro} y punto de control crítico (HCCP)

El sistema de Análisis de Peligro y Punto de Control Crítico (HACCP, por sus en inglés) que se basa en principios científicos y bajo un enfoque sistemático. Tiene como objeto identificar peligros específicos y las medidas que puedan aplicarse para controlar los peligros o riesgos detectados, esto en aras de garantizar la inocuidad de los alimentos. Este instrumento está diseñado para evaluar los peligros y establecer sistemas de control que se fundamentan en la prevención en lugar de las evaluaciones finales de la producción. Todo sistema de HACCP es susceptible a modificaciones que se derivan de cambios en los diseños del equipo, los procedimientos de elaboración o el sector técnico-tecnológico. El HACCP puede en todos los puntos de la cadena alimentaria y productiva, desde el productor primario hasta el consumidor final, y su aplicación se basa estrictamente en pruebas científicas de peligros para la salud humana, además de mejorar la calidad e inocuidad de los alimentos (Lorenzo, 2008).

Para que la aplicación del (HACCP) genere resultados positivos, se requiere que tanto la alta directiva como el personal se comprometan y participen activamente. Cabe señalar que la aplicación del sistema HACCP es compatible con los sistemas de gestión de la calidad basados en las normas ISO 9000 . Por otra parte, se constituye en el principal método para evaluar la inocuidad de los alimentos y la calidad de los alimentos en función de los requisitos propuestos según la norma ISO (Dzwolak, 2014).

\subsection{Definiciones}

Análisis de peligros: proceso de recopilación y evaluación de información sobre los peligros y las condiciones que los originan para decidir cuáles son importantes con la inocuidad de los alimentos $\mathrm{y}$, por tanto, planteados en el plan del sistema de Análisis de Peligro y Punto de Control Crítico (HACCP) (DeBeer et al., 2017). 
Controlado: condición obtenida por cumplimiento de los procedimientos y de los criterios marcados.

Controlar: adoptar todas las medidas necesarias para asegurar y mantener el cumplimiento de los criterios establecidos en el sistema de Análisis de Peligro y Punto de Control Crítico (HACCP).

Desviación: situación existente cuando un límite crítico es incumplido.

Diagrama de flujo: representación sistemática de la secuencia de fases u operaciones llevadas a cabo en la producción o elaboración de un determinado producto alimenticio.

Fase: cualquier punto, procedimiento, operación o etapa de la cadena alimentaria, incluidas las materias primas, desde la producción primaria hasta el consumo final.

Límite crítico: criterio que diferencia la aceptabilidad o inaceptabilidad del proceso en una determinada etapa.

Medida correctiva: son acciones que deben realizarse cuando los resultados de la vigilancia en los Puntos de Control Critico (PCC) indican fallos o pérdida en el control del proceso (DeBeer et al., 2017).

Medida de control: se trata de cualquier medida y actividad que puede realizarse para prevenir 0 eliminar un peligro para la inocuidad de los alimentos o para reducirlo a un nivel aceptable.

Peligro: son agentes biológicos, químicos o físicos presentes en el producto alimenticio, o en su defecto la condición en que éste se halla, que puede ocasionar consecuencias adversas para la salud.

Plan de HACCP (Análisis de Control y Punto de Control Crítico.): es un documento preparado de conformidad con los principios del sistema de HACCP, de manera que su cumplimiento garantiza el control de los peligros que resultan significativos para la inocuidad de los alimentos en el segmento de la cadena alimentaria.

Punto crítico de control (PCC): es la fase que debido a sus condiciones 0 características, puede aplicársele un control y que es esencial para prevenir, disminuir o eliminar un peligro asociado con la inocuidad y seguridad alimentaria. 
Sistema de Análisis de Peligro y Punto de Control Crítico (HACCP): es el sistema que permite identificar, evaluar y controlar peligros significativos para la inocuidad de los alimentos y consecuentemente garantizar la calidad de los alimentos.

Transparente: característica de un proceso cuya justificación, lógica de desarrollo, limitaciones, supuestos, juicios de valor, decisiones, limitaciones, e incertidumbres de la determinación alcanzada están explícitamente expresadas, documentadas y accesibles para su revisión.

Validación: consiste en la constatación de la efectividad de los elementos del plan de HACCP.

Verificación: es la aplicación de métodos, procedimientos, ensayos y otros mecanismos de evaluación, además de la vigilancia, para constatar el cumplimiento del plan de HACCP.

Vigilar: se basa en llevar a cabo una secuencia planificada de observaciones o mediciones de los parámetros de control para evaluar si un PCC se encuentra controlado (DeBeer et al., 2017).
La finalidad del sistema de HACCP es lograr que el control se centre en los PCC. En el caso de que se identifique un peligro que debe controlarse, pero no se encuentre ningún PCC, deberá considerarse la posibilidad de formular de nuevo la operación.

Cuando se introduzca alguna modificación en el producto, el proceso o en cualquier fase, será necesario examinar la aplicación del sistema de HACCP y realizar los cambios oportunos.

Finalmente, es de carácter importante que el sistema de HACCP se aplique de modo flexible, teniendo en cuenta el carácter y la amplitud de la operación (Pizarro, 2002).

\section{Recursos}

\subsection{Agua}

La contaminación de las aguas de superficie provenientes de las aguas residuales industriales y de aguas negras sin tratar es una de las causas principales de daños a la propiedad (en combinación con las inundaciones), pérdidas de espacios para recreación y daños ecológicos alrededor de las principales áreas urbanas y de varios lagos interiores. 
En varios lugares del mundo se han contaminado los recursos hídricos hasta el punto de afectar los trabajos de las plantas de tratamiento. Hay proyectos para hacer plantas de tratamiento para las principales localidades, pero la descarga sigue creciendo (Aurrichio, 2011). La contaminación del agua es la acción $y$ el efecto de introducir materias 0 formas de energía, o inducir condiciones en el agua que, de modo directo o indirecto, impliquen una alteración perjudicial de su calidad en relación con los usos posteriores - con su función ecológica (Aurrichio, 2001).

Los problemas del agua se centran tanto en la calidad como en la cantidad. La comunidad debe conocer la importancia de la "calidad" del agua y esa misma comunidad debe encargarse de su cuidado y preservación (ONU, 2015).

El agua es un recurso que encuentra disponible en muchos lugares, sin embargo, cerca de 1.100 millones de personas carecen de acceso al agua potable, a las que habría que sumar 2.400 millones de personas que no tienen acceso a un saneamiento adecuado de las aguas residuales (ONU, 2015).
La optimización de los recursos es una realidad latente en las áreas involucradas directamente con la elaboración del enlatado de atún, y es parte de la política de calidad de las empresas, de modo que toda empresa pesquera debe enfocarse en la mejora continua de sus procesos, desarrollando mecanismos para optimizar el uso de los recursos, principalmente el agua; todo esto sin alterar las condiciones de inocuidad en las cuales se basa la cada productiva alimentaria, desde la materia prima hasta el consumo del producto. En empresas atuneras con una tasa de producción diaria de se consumen entre 325 y $342 \mathrm{~m}^{3}$ de agua (Barclay \& Cartwright, 2007).

\section{Conclusiones}

La mejora permanente y renovadora de la calidad de los procesos productivos de las industrias pesqueras debe ser un principio que forme parte de la política de calidad, manejo ambiental y seguridad de los trabajadores. Sin embargo, la optimización de los procesos implica cambios en los procesos productivos, los cuales deben ser valorados en el sistema HACCP implantado en gran parte de las industrias pesqueras. 


\section{Bibliografía}

Aurrichio. (2001). Auditoría de Sistemas de Gestión Medioambiental. Introducción a la norma ISO 14001. Mc Graw Hill.

Barclay, K., \& Cartwright, I. (2007). Governance of tuna industries: The key to economic viability and sustainability in the Western and Central Pacific Ocean. Marine Policy, 31(3), 348-358.

DeBeer, J., Nolte, F., Lord, C. W., Colley, J., \& Weddig, L. (2017). Setting HACCP critical limits for the precooking CCP of commercially processed tuna. Food Protection Trends, 37(3), 176-188.

Dzwolak, W. (2014). HACCP in small food businesses-The Polish experience. Food control, 36(1), 132-137.

Lorenzo, L. C. (2008). Auditoría del sistema APPCC: Cómo verificar los sistemas de gestión de inocuidad alimentaria HACCP. Ediciones Díaz de Santos.

ONU. (2015). Objetivos de Desarrollo Sostenible. Organización de las Naciones Unidas. Ginebra.

Pizarro. (2002). Componentes y definiciones de un plan HACCP. El Sistema HACCP. Disponible en: www.conamype.gob.sv/biblio/ pdf/0033.pdf

Ruiz, A., \& Karim, G. (2016). Optimización de las operaciones en la fase primaria mediante el análisis de los procesos. Instituto Tecnológico de Colima. 